\title{
Generalized thermostatistics based on deformed exponential and logarithmic functions
}

\author{
Jan Naudts \\ Departement Natuurkunde, Universiteit Antwerpen, \\ Universiteitsplein 1, 2610 Antwerpen, Belgium \\ E-mail Jan.Naudts@ua.ac.be
}

\begin{abstract}
The equipartition theorem states that inverse temperature equals the log-derivative of the density of states. This relation can be generalized by introducing a proportionality factor involving an increasing positive function $\phi(x)$. It is shown that this assumption leads to an equilibrium distribution of the Boltzmann-Gibbs form with the exponential function replaced by a deformed exponential function. In this way one obtains a formalism of generalized thermostatistics introduced previously by the author. It is shown that Tsallis' thermostatistics, with a slight modification, is the most obvious example of this formalism and corresponds with the choice $\phi(x)=x^{q}$.
\end{abstract}

Key words: Generalized thermostatistics, equipartition theorem, density of states, deformed logarithmic and exponential functions, Tsallis' thermostatistics, duality

\section{What is thermostatistics?}

See [1] for a conceptual foundation of thermostatistics. The presentation here emphasizes the role of the density of states.

A model of thermostatistics is described by a density of states $\rho(E)$ and a probability distribution $p(E)$, both functions of energy $E$. For a system in thermal equilibrium at temperature $T$, the probability distribution is given by the Boltzmann-Gibbs expression

$$
\begin{aligned}
& p(E)=\frac{1}{Z(T)} e^{-E / T} \quad \text { with } \\
& Z(T)=\int \mathrm{d} E \rho(E) e^{-E / T}
\end{aligned}
$$


(Boltzmann's constant $k_{B}$ is set equal to 1 ). Thermal averages are defined by

$$
\langle f\rangle=\int \mathrm{d} E \rho(E) p(E) f(E) .
$$

Conserved quantities, other than energy, might be important. It is tradition to give a simplified treatment involving only energy. To keep notations simple the temperature dependence of $p(E)$ is not made explicit: $p(E)$ stands for $p(E, T)$.

A microscopic model of thermostatistics is specified by an energy functional $H(\gamma)$ over phase space $\Gamma$, which is the set of all possible microstates of the system. Using $\rho(E) \mathrm{d} E=\mathrm{d} \gamma$ the integration in expressions $(2,3)$ can be replaced by an integration over phase space

$$
\begin{aligned}
\langle f\rangle & =\int_{\Gamma} \mathrm{d} \gamma p(\gamma) f(\gamma) \quad \text { with } \\
p(\gamma) & =\frac{1}{Z(T)} \exp (-H(\gamma) / T) \quad \text { and } \\
Z(T) & =\int_{\Gamma} \mathrm{d} \gamma \exp (-H(\gamma) / T) .
\end{aligned}
$$

In the quantum case the integration is replaced by a trace over operators. The formulas are

$$
\begin{aligned}
\langle f\rangle & =\frac{1}{Z(T)} \operatorname{Tr} \exp (-H / T) f \quad \text { with } \\
Z(T) & =\operatorname{Tr} \exp (-H / T)
\end{aligned}
$$

Paraphrasing the words of $\mathrm{C}$. Tsallis during this conference, one can state that the Boltzmann-Gibbs distribution is not just an exponential distribution, but that it is important that it is a sum over all states of phase space, and that the exponential contains energy divided by temperature. In particular, if a microscopic model reproduces the experimentally observed probabilities $p(\gamma)$ at one given temperature then one can predict their value at all other temperatures. This predictive power is the main asset of thermostatistics.

\section{Why Boltzmann-Gibbs?}

In relevant examples of thermostatistics the density of states $\rho(E)$ increases as a power law $\rho(E) \sim E^{\alpha N}$ with $N$ the number of particles and with $\alpha>0$. 
This increase in the density of states is essential to understand the paradox that according to the Boltzmann-Gibbs distribution the ground state is always the most probable state. Still, one never observes that the molecules of the air in a class room lie all on the floor. Moreover, energy fluctuations of a gas in equilibrium, away from any phase transition temperature, are negligible. The solution to the paradox is known as the entropy-energy balance. The increase of density of states $\rho(E)$ compensates the exponential decrease of probability density $p(E)$. The maximum of the product $\rho(E) p(E)$ is reached at some macroscopic energy far above the ground state energy. Indeed, one can write

$$
\rho(E) p(E)=\frac{1}{Z(T)} \exp (\log \rho(E)-E / T)
$$

The argument of the exponential function is maximal if $E$ satisfies

$$
\frac{1}{\rho(E)} \rho^{\prime}(E)=\frac{1}{T}
$$

$\left(\rho^{\prime}(E)\right.$ is the derivative of $\rho(E)$ w.r.t. $\left.E\right)$. If $\rho(E) \sim E^{\alpha N}$ then $E \simeq \alpha N T$ follows, which is the equipartition theorem.

As a consequence of the equipartition theorem it is not easy to verify the Boltzmann-Gibbs distribution experimentally. Indeed, the energy of the system under study is always equal to the value predicted by (10), with negligible fluctuations. This indicates that the actual form of the probability distribution $p(E)$ is not firmly established and, in fact, is not very essential. Alternative expressions for $p(E)$ are acceptable provided they satisfy the equipartition theorem and reproduce thermodynamics. An indication of the need for a generalization of Boltzmann-Gibbs is the ubiquitous use of temperature-dependent Hamiltonians in applied statistical physics. As stressed in the previous section, (1) predicts the probability density $p(E)$ at all temperatures. In many cases this prediction is not very accurate, probably because of an incomplete knowledge of the density of states $\rho(E)$. However, instead of making $\rho(E)$ temperature-dependent, which is not supported by theory, one can as well try to replace the Boltzmann-Gibbs distribution by another expression more appropriate for the problem at hand.

\section{The basic postulate}

The present generalization of thermostatistics starts with generalizing the equipartition result (10). Let us postulate the existence of an increasing positive function $\phi(x)$, defined for $x \geq 0$, such that 


$$
\frac{1}{T}=\frac{-p^{\prime}(E)}{\phi(p(E))}
$$

holds for all energies $E$ and temperatures $T$. Then the equation for the maximum of $\rho(E) p(E)$ becomes

$$
\begin{aligned}
0 & =\frac{\mathrm{d}}{\mathrm{d} E}(\rho(E) p(E)) \\
& =\rho^{\prime}(E) p(E)-\frac{1}{T} \rho(E) \phi(p(E)) .
\end{aligned}
$$

This can be written as

$$
\frac{\rho^{\prime}(E)}{\rho(E)}=\frac{1}{T} \frac{\phi(p(E))}{p(E)}
$$

The latter expression generalizes the equipartition theorem (10). The Boltzmann-Gibbs case is recovered with $\phi(x)=x$.

The postulate (11) fixes the form of the probability distribution $p(E)$. To see this, introduce a function, denoted $\ln _{\phi}(x)$, by

$$
\ln _{\phi}(x)=\int_{1}^{x} \mathrm{~d} y \frac{1}{\phi(y)} .
$$

There are good reasons for calling this function a deformed logarithm. If $\phi(x)=x$ then it coincides with the natural logarithm $\log (x)$. Because $\phi(x)$ is positive for all positive $x$ one has that $\ln _{\phi}(x)$ is negative for $0<x<1$ and positive for $x>1$. With some further technical conditions this function becomes a deformed logarithm in the sense of [2].

The inverse of the function $\ln _{\phi}(x)$ is denoted $\exp _{\phi}(x)$. From the identity $1=$ $\exp _{\phi}^{\prime}\left(\ln _{\phi}(x)\right) \ln _{\phi}^{\prime}(x)$ follows

$$
\phi(x)=\exp _{\phi}^{\prime}\left(\ln _{\phi}(x)\right) .
$$

Hence (11) can be written as

$$
p^{\prime}(E)=-\frac{1}{T} \exp _{\phi}^{\prime}\left[\ln _{\phi}(p(E))\right] .
$$

This expression can be integrated. The result is

$$
p(E)=\exp _{\phi}\left(G_{\phi}(T)-E / T\right) .
$$


The function $G_{\phi}(T)$ is the integration constant. It must be chosen in such a way that the normalization condition

$$
1=\int \mathrm{d} E \rho(E) p(E)
$$

is satisfied. Positivity $p(E) \geq 0$ is automatic because the range of the function $\exp _{\phi}(x)$ is the domain of $\ln _{\phi}(x)$, with possibly 0 and $+\infty$ added. Expression (17) resembles the Boltzmann-Gibbs distribution (1). An important difference is that the normalization constant appears inside the function $\exp _{\phi}(x)$. In case $\phi(x)=x$ then one has $G_{\phi}(T)=-\log (Z(T))$.

Starting from (17) a generalized thermostatistics can be developed — see [3]. Most of the results that follow below are reformulations of the results found in the first part of [3]. The variational principle obeyed by (17) is not discussed below.

\section{Escort probabilities}

In general it is difficult to calculate the integration constant $G_{\phi}(T)$. A useful expression for its temperature derivative can be obtained in terms of escort probabilities. They originate from [4] and have been introduced in Tsallis' thermostatistics in [5]. The generalized definition is

$$
P(E)=\frac{1}{Z(T)} \phi(p(E))
$$

with normalization factor

$$
Z(T)=\int \mathrm{d} E \rho(E) \phi(p(E))
$$

Expectation values w.r.t. $P(E)$ are denoted

$$
\langle f\rangle_{*}=\int \mathrm{d} E \rho(E) P(E) f(E) .
$$

Note that $P(E)$ coincides with $p(E)$ in the Boltzmann-Gibbs case $\phi(x)=x$ for all $x$.

Now calculate, using (15) and (19), 


$$
\begin{aligned}
\frac{\mathrm{d}}{\mathrm{d} T} p(E) & =\exp _{\phi}^{\prime}\left(G_{\phi}(T)-E / T\right)\left(\frac{\mathrm{d}}{\mathrm{d} T} G_{\phi}(T)+\frac{E}{T^{2}}\right) \\
& =Z(T) P(E)\left(\frac{\mathrm{d}}{\mathrm{d} T} G_{\phi}(T)+\frac{E}{T^{2}}\right)
\end{aligned}
$$

From (18) and (22) follows

$$
\begin{aligned}
0 & =\int \mathrm{d} E \rho(E) \frac{\mathrm{d}}{\mathrm{d} T} p(E) \\
& =Z(T) \frac{\mathrm{d}}{\mathrm{d} T} G_{\phi}(T)+\frac{1}{T^{2}} Z(T)\langle E\rangle_{*} .
\end{aligned}
$$

Hence one has

$$
\frac{\mathrm{d}}{\mathrm{d} T} G_{\phi}(T)=-\frac{1}{T^{2}}\langle E\rangle_{*}
$$

For further use note that (22) and (24) together give

$$
\frac{\mathrm{d}}{\mathrm{d} T} p(E)=\frac{1}{T^{2}} Z(T) P(E)\left(E-\langle E\rangle_{*}\right)
$$

\section{Thermodynamic relations}

One goal of thermostatistics is to give a microscopic derivation of the laws of thermodynamics. This raises immediately the question in how far generalized thermostatistics is still compatible with thermodynamics.

Let us start with establishing thermal stability. Internal energy $U(T)$ is defined by $U(T)=\langle E\rangle$, with $p(E)$ given by (17). Using (25) one obtains

$$
\begin{aligned}
\frac{\mathrm{d}}{\mathrm{d} T} U(T) & =\int \mathrm{d} E \rho(E) E \frac{\mathrm{d}}{\mathrm{d} T} p(E) \\
& =\int \mathrm{d} E \rho(E) E \frac{1}{T^{2}} Z(T) P(E)\left(E-\langle E\rangle_{*}\right) \\
& =\frac{1}{T^{2}} Z(T)\left(\left\langle E^{2}\right\rangle_{*}-\langle E\rangle_{*}^{2}\right) \\
& \geq 0
\end{aligned}
$$

Hence, average energy is an increasing function of temperature. However, thermal stability requires more. Define $\phi$-entropy (relative to $\rho(E) \mathrm{d} E$ ) by 


$$
S_{\phi}(p)=\int \mathrm{d} E \rho(E)\left[(1-p(E)) F_{\phi}(0)-F_{\phi}(p(E))\right]
$$

with

$$
F_{\phi}(x)=\int_{1}^{x} \mathrm{~d} y \ln _{\phi}(y) .
$$

Let us postulate that thermodynamic entropy $S(T)$ equals the value of the above entropy functional $S_{\phi}(p)$ with $p$ given by (17). Then one finds

$$
\begin{aligned}
\frac{\mathrm{d}}{\mathrm{d} T} S(T) & =\int \mathrm{d} E \rho(E)\left(-\ln _{\phi}(p(E))-F_{\phi}(0)\right) \frac{\mathrm{d}}{\mathrm{d} T} p(E) \\
& =\int \mathrm{d} E \rho(E)\left(-G_{\phi}(T)+\frac{E}{T}-F_{\phi}(0)\right) \frac{\mathrm{d}}{\mathrm{d} T} p(E) \\
& =\frac{1}{T} \frac{\mathrm{d}}{\mathrm{d} T} U(T)
\end{aligned}
$$

To see the latter use that $p(E)$ is normalized to 1 . This shows that temperature $T$ satisfies the thermodynamic relation

$$
\frac{1}{T}=\frac{\mathrm{d} S}{\mathrm{~d} U}
$$

Because energy is an increasing function of temperature one concludes that entropy $S$ is a concave function of energy $U$. This property is known as thermal stability.

One can introduce the Helmholtz free energy $F(T)$ by the well-known relation $F(T)=U(T)-T S(T)$. From (29) follows that

$$
\frac{\mathrm{d}}{\mathrm{d} \beta} \beta F(T)=U(T) \quad \text { with } \beta=\frac{1}{T}
$$

Now let us come back to (24), which is very similar to (31), with $F(T)$ replaced by $T G_{\phi}(T)$ and with $U(T)=\langle E\rangle$ replaced by $\langle E\rangle_{*}$. The comparison shows that $T G_{\phi}(T)$ equals the free energy associated with the escort probability distribution $P(E)$, up to a constant independent of temperature $T$.

\section{Tsallis' thermostatistics}

The most obvious generalization of Boltzmann-Gibbs thermostatistics is obtained by the choice $\phi(x)=x^{q}$ with $q>0$. It reproduces Tsallis' thermostatis- 
tics with some minor changes. The corresponding deformed logarithmic and exponential functions are

$$
\begin{aligned}
\ln _{q}(x) & =\int_{1}^{x} \mathrm{~d} y y^{-q}=\frac{1}{1-q}\left(x^{1-q}-1\right) \\
\exp _{q}(x) & =[1+(1-q) x]_{+}^{1 /(1-q)} .
\end{aligned}
$$

These have been introduced in [6]. The probability distribution (17) becomes

$$
\begin{aligned}
p(E) & =\left[1+(1-q)\left(G_{q}(T)-E / T\right)\right]_{+}^{1 /(1-q)} \\
& =\frac{1}{z_{q}(T)}\left[1-(1-q) \beta_{q}^{*}(T) E\right]_{+}^{1 /(1-q)}
\end{aligned}
$$

with

$$
\begin{aligned}
z_{q}(T) & =\left(1+(1-q) G_{q}(T)\right)^{1 /(q-1)} \quad \text { and } \\
\beta_{q}^{*}(T) & =z_{q}(T)^{1-q} / T .
\end{aligned}
$$

Originally [7], (34) was introduced with $q$ replaced by $2-q$. The present expression was proposed in [8], be it with a different expression for $\beta_{q}^{*}(T)$.

A nice feature of Tsallis' thermostatistics is that the correspondence between $p(E)$ and the escort $P(E)$ leads to a dual structure, called the ' $q \leftrightarrow 1 / q^{\prime}-$ duality $[5,9]$. Indeed, from

$$
P(E)=\frac{1}{Z_{q}(T)} p(E)^{q} \quad \text { follows } \quad p(E)=\frac{1}{Z_{1 / q}(T)} P(E)^{1 / q} .
$$

However, there exists also a ' $q \leftrightarrow 2-q$ '-duality the origin of which has been clarified in [2]. Given $\ln _{\phi}(x)$, a new deformed logarithmic function $\ln _{\psi}(x)$ is obtained by

$$
\ln _{\psi}(x)=(x-1) F_{\phi}(0)-x F_{\phi}(1 / x),
$$

with $\psi(x)$ given by

$$
\frac{1}{\psi(x)}=F_{\phi}(0)-F_{\phi}(1 / x)+\frac{1}{x} \ln _{\phi}\left(\frac{1}{x}\right)
$$

In case $\phi(x)=x^{q}$ follows $\psi(x)=(2-q) x^{2-q}$. Hence, the deformed logarithms $\ln _{q}(x)$ and $\ln _{2-q}(x)$ can be deduced from each other, up to a constant factor, by the relation $(37)$. 
Now note that the definition $(27)$ of entropy $S_{\phi}(p)$ can be written as

$$
S_{\phi}(p)=\int \mathrm{d} E \rho(E) p(E) \ln _{\psi}(1 / p(E)) .
$$

With $\psi(x)=x^{q}$ this expression is Tsallis' entropy

$$
S_{q}(p)=\int \mathrm{d} E \rho(E) \frac{1}{1-q}\left(p(E)^{q}-p(E)\right) .
$$

However, as noted above, $\psi(x) \sim x^{2-q}$ is needed. This leads to an expression for entropy with $q$ replaced by $2-q$. In the Tsallis literature one has preferred to use always the same expression (40) for the entropy functional. Instead, the definition of average energy has been changed [5] from $\langle E\rangle$ to $\langle E\rangle_{*}$. For most physicists the latter change is unacceptable. During this conference $\mathrm{F}$. Baldovin suggested to interchange the roles of $p(E)$ and $P(E)$ so that the need for changing the definition of average energy disappears. This is what happened in the present paper.

\section{Conclusions}

It is possible to formulate a generalized thermostatistics in which the equipartition theorem holds in a modified form. Some aspects of thermodynamics, like thermal stability, are recovered. Other aspects, like extensivity, have not been considered because they will not hold in the generalized context. In the presentation, the role of the density of states $\rho(E)$ has been emphasized. By doing so the link is made between probability distribution functions $p(E)$ depending on energy $E$ and the distributions $p(\gamma)$ depending on points $\gamma$ in phase space $\Gamma$.

The generalized formalism is determined by the choice of an increasing positive function $\phi(x)$, defined for $x \geq 0$. The standard formalism corresponds with $\phi(x)=x$ for all $x$. Tsallis' thermostatistics, with some minor changes, corresponds with the obvious choice $\phi(x)=x^{q}$. The function $\phi(x)$ can be used to define deformed logarithmic and exponential functions. The equilibrium probability distribution function $p(E)$ is then a generalization of the BoltzmannGibbs distribution, obtained by replacing the exponential function $\exp (x)$ by the deformed exponential function.

One of the advantages of formulating a rather general theory is that it clarifies some aspects of Tsallis' thermostatistics by putting them in a broader context. In particular, the ' $q \leftrightarrow 1 / q$ ' and ' $q \leftrightarrow 2-q$ '-dualities have been discussed. The former one concerns the role of escort probabilities. The latter concerns 
the observation that from each deformed logarithm one can deduce another deformed logarithm. Clarifying these points makes clear that there is no need to modify the definition of average energy, as has been done in the recent Tsallis literature. Rather one should replace Tsallis' entropy $S_{q}(p)$ by $S_{2-q}(p)$. In this way one obtains a formalism which is satisfactory both from a physical and from a mathematical point of view, and which can be generalized to a much broader scope when needed.

\section{References}

[1] H.B. Callen, Thermodynamics and an introduction to thermostatistics, 2nd edition (John Wiley \& Sons, 1985)

[2] J. Naudts, Deformed exponentials and logarithms in generalized thermostatistics, arXiv:cond-mat/0203489, Physica A316, 1-12 (2002).

[3] J. Naudts, Generalized thermostatistics and mean field theory, arXiv:cond-mat/0311444v6, to appear in Physica A.

[4] C. Beck, F. Schlögl, Thermodynamics of chaotic systems: An introduction (Cambridge University Press, Cambridge, 1993)

[5] C. Tsallis, R.S. Mendes, A.R. Plastino, The role of constraints within generalized nonextensive statistics, Physica A261, 543-554 (1998).

[6] C. Tsallis, What are the numbers that experiments provide? Quimica Nova 17, 468 (1994).

[7] C. Tsallis, Possible Generalization of Boltzmann-Gibbs Statistics, J. Stat. Phys. 52, 479-487 (1988).

[8] E.M.F. Curado, C. Tsallis, Generalized statistical mechanics: connection with thermodynamics, J. Phys. A 24, L69-72 (1991).

[9] J. Naudts, Dual description of nonextensive ensembles, arXiv:cond-mat/9904070v2, Chaos, Solitons, and Fractals, 13(3), 445-450 (2002). 